\title{
Oferta de cuidado primário para doenças crônicas: uma análise da eficiência técnicas das equipes de saúde brasileiras
}

\author{
Thiago Augusto Hernandes Rocha, Núbia Cristina da Silva.
}

\section{RESUMO}

Introdução: Este trabalho, portanto, tem como objetivo analisar comparativamente a eficiência de equipes de Saúde da Família dos diferentes estados brasileiros quanto às ações de prevenção, tratamento e acompanhamento da hipertensão arterial e do diabetes mellitus. A partir do resultado será possível traçar as ações que devem ser priorizadas, para que as equipes se tornem eficientes nos processos fundamentais para 0 cuidado relativo a estas doenças.

Método: A avaliação da eficiência das equipes de Saúde da Família foi elaborada a partir da metodologia DEA (Data Envelopment Analysis). DEA que utiliza a técnica de programação linear para cálculo da eficiência relativa de um conjunto de unidades produtivas que possuem função comum e operem de forma similar, mas cuja eficiência varia em função de aspectos internos. Para este trabalho optou-se pelo modelo CRS orientado a outputs para avaliar a eficiência das equipes de saúde da família (DMU's). Ao todo foram avaliadas 2133 equipes de saúde da família, amostra está definida com um

Revista da Rede APS 2019

Publicada em:

$22 / 03 / 2019$

Thiago Augusto Hernandes Rocha (OPAS/DF), Núbia Cristina da Silva (M.A.T.H Consortium)

Correspondência para: Thiago Augusto Hernandes Rocha, rochahernandes3@gmail. com. erro máximo de $2 \%$.

Resultados: A maior parte dos estados possui entre $5 \%$ e $15 \%$ de equipes eficientes, percentual que pode ser considerado baixo e que mantém relação com a qualidade dos serviços de saúde dedicados a atender condições crônicas, frente aos insumos disponíveis. A grande dispersão dos percentuais de eficiência nos estados evidencia a heterogeneidade nas regiões

Conclusões: Por fim, entende-se que o aumento da eficiência neste caso, além de possibilitar melhor oferta de cuidados à população, representa eficiência na utilização dos recursos materiais e financeiros, o que se torna imprescindível no contexto das políticas públicas. Neste sentido, vale destacar que as ações de intervenção no nível da atenção primária impactam de forma expressiva o sistema de saúde como um todo, pois evitam agravos que sobrecarregam os demais níveis de atenção nos quais há maior necessidade de recursos e, por isso, maiores custos, além de maior risco para os pacientes. Assim, os resultados obtidos com a utilização da metodologia de DEA assumem grande relevância ao ressaltar a necessidade de maior investimento para que este nível de atenção à saúde cumpra seu papel na organização da rede de saúde.

Palavras-chave: Pesquisa Operacional, Assistência à Saúde, Doença crônica. 


\section{INTRODUÇÃO}

Desde meados da década de 40 a configuração populacional brasileira vem sofrendo alterações significativas. Entre os anos 40 e 60, um estudo elaborado pelo IBGE (principal provedor de dados e informações do país, que atendem às necessidades dos mais diversos segmentos da sociedade civil, bem como dos órgãos governamentais) destacou que o Brasil experimentou um declínio significativo da mortalidade, mantendo a taxa fecundidade em níveis elevados, o que produziu uma população quase-estável jovem e com rápido crescimento. Ao final da década de 60 , com a redução da fecundidade generalizada entre os diversos grupos etários, iniciou-se um processo de transição etária para um perfil populacional envelhecido e com baixo ritmo de crescimento (1).

Além do envelhecimento, observou-se nos últimos 50 anos uma modificação do perfil epidemiológico da população (2). Esta transformação é definida por Omran (3) como transição epidemiológica, e é marcada pela evolução progressiva de um perfil de alta mortalidade por doenças infecciosas para outro no qual predominam os óbitos por doenças cardiovasculares, neoplasias, causas externas e outras doenças crônico-degenerativas. No entanto, considera-se que no Brasil a presença das doenças infecciosas é ainda significativa e, combinada ao aumento da incidência das doenças crônicas não transmissíveis (DCNT), caracterizam a situação de dupla carga de doenças, a qual exige políticas específicas para o cuidado da população (4).

A Organização Mundial da Saúde (OMS) define como doenças crônicas as cardiovasculares (cerebrovasculares, isquêmicas), neoplasias, doenças respiratórias crônicas e o diabetes mellitus (5). A OMS também inclui nesse rol aquelas doenças que contribuem para o sofrimento dos indivíduos, das famílias e da sociedade, tais como as desordens mentais e neurológicas, entre outras. No Brasil, a abordagem tem sido focada no diabetes e na hipertensão, pois manifestam os principais fatores de risco das doenças cardiovasculares, as quais constituem a principal causa de morbimortalidade da população brasileira (6).
A forma considerada mais adequada para organização dos serviços de saúde capaz de manejar efetivamente a dupla carga de adoecimento perpassa por uma atenção primária fortalecida e resolutiva. Isso porque os sistemas com orientação para a atenção primária à saúde são considerados mais adequados às necessidades de saúde da população; mais eficientes; apresentam menores custos; de maior qualidade para os pacientes; e mais efetivos para enfrentar a situação epidemiológica de crescimento das condições crônicas $(7,8)$.

As complicações em decorrência do diabetes mellitus e da hipertensão arterial, especificamente, afetam de modo substancial a expectativa e a qualidade de vida dos brasileiros. A expressão clínica de ambas se dá após longo tempo de exposição aos fatores de risco, além da convivência assintomática do indivíduo com a doença não diagnosticada, mesmo quando os fatores de risco são perceptíveis (tabagismo, obesidade generalizada, obesidade central com ou sem obesidade generalizada, alcoolismo, sedentarismo, etc). Consequentemente, os diagnósticos podem ocorrer em fases tardias, com a doença já complicada ou num episódio que pode ser o primeiro e fatal, como ocorre muitas vezes com a doença coronariana aguda e com o acidente vascular encefálico (6). Graças às semelhanças entre os fatores de risco de ambas é possível contar com uma abordagem comum para sua prevenção.

O tratamento das doenças crônicas em serviços de urgência, emergência ou hospitalizações, além de mais oneroso, não favorece as mudanças desejáveis no sistema de saúde, pois não contribui para a prática da promoção e proteção da saúde com prevenção de agravos, tendo um enfoque apenas curativo. Com base nestes dados, tem-se preconizado mudanças nos sistemas nacionais de saúde, para que se voltem para um modelo de atenção contínua centrado na atenção primária, e não somente organizado para o cuidado de condições predominantemente agudas $(8,9,10$, 11).

Entende-se, a partir disso, que o sistema de saúde deve estar organizado e preparado para lidar com estas características. O Ministério da Saúde no 
Brasil, baseado em evidências na área e em experiências de outros países, destaca que as tecnologias mais efetivas para a redução da carga de doenças crônicas são aquelas que trabalham com os fatores determinantes e condicionantes da saúde individual e coletiva, elaborando políticas públicas orientadas pela ótica do cuidado integral à saúde (11).

A concretização de um sistema centrado na atenção primária à saúde (APS) no Brasil, no qual os níveis de atenção se organizam em formato de rede e a APS ocupa o centro desta rede, se deu por meio da implementação do Programa Saúde da Família (PSF) em 1994. O programa funciona em unidades básicas de saúde, ofertando cuidado por meio de uma equipe composta por: médico, enfermeiro, técnico de enfermagem e agentes comunitários de saúde. Essas equipes atendem a uma clientela populacional adscrita à unidade de saúde de modo a fornecer cuidado contínuo, tendo por base o estabelecimento de um vínculo de cuidado entre população e profissionais $(12,13)$.

Nesta linha, a Saúde da Família no Brasil deve atuar segundo as políticas sanitárias definidas para estas doenças. Por isso, para atenção aos diabéticos e hipertensos é necessário que sejam utilizadas tecnologias orientadas e preparadas para a abordagem de processos de adoecimento de mais longo prazo e de causalidade complexa, para os quais se articulam diferentes fatores individuais, coletivos, sociopolíticos, culturais, históricos e econômicos (11).

Este trabalho, portanto, tem como objetivo analisar comparativamente a eficiência de equipes de Saúde da Família dos diferentes estados brasileiros quanto às ações de prevenção, tratamento e acompanhamento da hipertensão arterial e do diabetes mellitus. A partir do resultado será possível traçar as ações que devem ser priorizadas, para que as equipes se tornem eficientes nos processos fundamentais para o cuidado relativo a estas doenças

\section{MÉTOdos}

Neste estudo, a avaliação da eficiência das equipes de Saúde da Família foi elaborada a partir da metodologia DEA (Data Envelopment Analysis), modelo desenvolvido inicialmente por Charnes, Cooper e Rhodes, em 1978. DEA que utiliza a técnica de programação linear para cálculo da eficiência relativa de um conjunto de unidades produtivas que possuem função comum e operem de forma similar, mas cuja eficiência varia em função de aspectos internos (14).

Esta técnica permite que problemas com múltiplos recursos (inputs) e múltiplas saídas (outputs) sejam resolvidos a partir da determinação da eficiência de cada Decision Making Unit (DMU). A eficiência é medida comparando-se as produtividades de cada unidade com a máxima produtividade observada no grupo analisado. O máximo que foi produzido para cada nível de recursos compõe a fronteira de eficiência, na qual estão localizadas as unidades com maior produtividade $(14,15)$.

Existem dois modelos básicos para análise de eficiência por meio de DEA, o CRS e o VRS. O modelo CRS (Constant Returns to Scale) trabalha com retornos constantes de escala, isto é, considera que qualquer variação nos inputs produz variação proporcional nos outputs. O modelo VRS (Variable Returns to Scale), ao contrário, não considera a proporcionalidade entre inputs e outputs e assume retornos variáveis (16).

Para este trabalho optou-se pelo modelo CRS orientado a outputs para avaliar a eficiência das equipes de saúde da família (DMU's), corroborando a premissa de que a oferta de cuidados de saúde a diabéticos e hipertensos aumenta proporcionalmente ao aumento de equipamentos, materiais, medicamentos e profissionais de saúde. Além disso, o modelo CRS foi utilizado em função da ausência de evidências referentes à existência de economia de escala na APS (17). A orientação a outputs veio da concepção de que as equipes de saúde da família precisam entregar a maior quantidade possível de serviços em saúde dado o suporte com o qual contam (inputs) para o desempenho de suas atividades.

$\mathrm{Na}$ abordagem da eficiência das equipes quanto à atenção ao diabetes e hipertensão, foram utilizados dados coletados junto à pesquisa de monitoramento nacional da Saúde da Família sobre 
estrutura, processo de trabalho e recursos humanos. Esta pesquisa foi conduzida no segundo semestre de 2008 pelo Observatório de Recursos Humanos em Saúde da Faculdade de Ciências Econômicas da Universidade Federal de Minas Gerais em parceria com o Ministério da Saúde e da Organização Pan-Americana de Saúde. A amostra da pesquisa foi selecionada de modo a obter representatividade por estado levando em conta dois parâmetros populacionais: porte (número de habitantes) e índice de desenvolvimento humano (IDH). Ao todo foram avaliadas 2133 equipes de saúde da família, amostra está definida com um erro máximo de $2 \%$.

A escolha dos inputs e outputs considera o modelo conceitual para avaliação em saúde proposto por Donabedian (18), o qual define a avaliação de ações de saúde a partir de três dimensões: estrutura, processo e resultado. Neste contexto, a avaliação da estrutura, mostra-se importante na medida em que a disponibilidade de materiais, insumos e equipamentos pode influenciar todo 0 desenvolvimento dos processos no serviço de saúde e, por consequência, causar impacto nos resultados. A avaliação dos processos, por sua vez, é considerada o caminho mais direto para o exame da qualidade do cuidado ofertado, tendo em vista as especificidades do setor de saúde, ao passo que os resultados, por refletirem os efeitos de todos os insumos do cuidado, são indicadores de qualidade tanto da estrutura quanto dos processos (19).

Assim, as abordagens de estrutura, processo e resultado guardam relação entre si, ou seja, assim como a estrutura influencia os processos, estes últimos são condicionantes dos resultados observados (18). Embora essa relação seja apenas probabilística e não de certeza, a avaliação destas três dimensões torna possível a construção de inferências sobre a qualidade dos serviços de saúde. Neste sentido, optou-se pela construção de indicadores que refletissem o modelo de avaliação proposto por Donabedian (sem considerar a dimensão de resultados que extrapola as ações da equipe, e, portanto, não pode ser tratada com a metodologia de $D E A$ ), tanto para a abordagem do diabetes, quanto da hipertensão arterial, os quais são apresentados no quadro abaixo:

Para este trabalho foram excluídas aquelas equipes que apresentavam dados faltantes em relação aos insumos e produtos avaliados para o cuidado em diabetes e hipertensão. Após esta seleção inicial foram analisadas 1522 equipes de saúde quanto aos cuidados de diabetes e 1532 quanto aos cuidados de hipertensão.

Os dados foram agregados segundo os indicadores acima para cada uma das equipes avaliadas. A eficiência relativa se deu em função da parcela de cumprimento do processo de trabalho para cada doença crônica em função dos insumos existentes, sendo que o índice de eficiência remete aos resultados obtidos pelas equipes segundo o modelo de avaliação de Donabedian (18). A despeito do fato da atenção em saúde ser uma prática multideterminada, pode-se afirmar que os outputs selecionados mantêm uma relação intrínseca com os inputs, o que confere validade ao modelo proposto.

Ainda sobre os indicadores, foi imposta ao modelo a restrição de que a variável recursos humanos não poderia ter peso igual a zero, considerando o fato de que a utilização intensiva de mão-de-obra é uma característica do serviço de saúde e, por isso, a oferta de cuidado sem a existência de profissionais de saúde é inviável.

Por fim, os índices de eficiência obtidos foram gerados para cada DMU do modelo. Para fins de apresentação dos dados, no entanto, estão descritos de forma sintetizada por valor médio, mínimo e máximo do estado e/ou da região administrativa do Brasil. Todas as análises foram feitas utilizando o software Frontier Analyst@ $@$. 
Quadro 1: Detalhamento de insumos e produtos

\begin{tabular}{|c|c|c|}
\hline Inputs & \multicolumn{2}{|c|}{ Definição } \\
\hline Atendimento & \multicolumn{2}{|c|}{ Tamanho da população atendida por cada uma das equipes de saúde. } \\
\hline Medicamentos & \multicolumn{2}{|c|}{$\begin{array}{l}\text { Percentual de medicamentos disponíveis para oferta de cuidado ao diabetes e } \\
\text { hipertensão, em relação ao que é disponibilizado pelo Ministério da Saúde. }\end{array}$} \\
\hline & \multicolumn{2}{|c|}{$\begin{array}{l}\text { Percentual de materiais e insumos existentes nas unidades de saúde, frente à } \\
\text { listagem abaixo. }\end{array}$} \\
\hline & Diabetes Mellitus & Hipertensão arterial \\
\hline Materiais e insumos & $\begin{array}{l}\text { - } \text { Glicosímetro } \\
\text { - } \text { medadeira para armazenar } \\
\text { - } \text { Fita HGT (glicemia capilar) } \\
\text { - } \quad \text { Lanceta para punção digital } \\
\text { - } \quad \text { Seringas com agulha acoplada } \\
\text { - } \text { para insulina } \\
\text { monitoramento de sistema de } \\
\text { monitoramento }\end{array}$ & $\begin{array}{l}\text { - Esfigmomanômetro } \\
\text { - Estetoscópio } \\
\text { - } \text { Balança para adultos } \\
\text { - Fita métrica } \\
\text { - Formulários de } \\
\text { monitoramento }\end{array}$ \\
\hline Recursos Humanos & \multicolumn{2}{|c|}{$\begin{array}{l}\text { Quantidade de profissionais existentes na equipe frente à equipe mínima } \\
\text { (médico, enfermeiro, agente de saúde e técnico de enfermagem). }\end{array}$} \\
\hline Indicadores de Outputs & \multicolumn{2}{|c|}{ Definição } \\
\hline Diagnóstico & \multicolumn{2}{|c|}{ Realização de consulta para diagnóstico de hipertensão e diabetes. } \\
\hline Acompanhamento & \multicolumn{2}{|c|}{$\begin{array}{l}\text { Desenvolvimento de ações para acompanhamento de ambas as doenças com } \\
\text { prescrição de medicamentos, sendo que para diabetes deve ser realizada, } \\
\text { também, a glicemia capilar para assintomáticos. }\end{array}$} \\
\hline Cadastro & \multicolumn{2}{|c|}{$\begin{array}{l}\text { Cadastro dos dados dos pacientes em sistemas de monitoramento e } \\
\text { acompanhamento. }\end{array}$} \\
\hline
\end{tabular}

Fonte: elaborado pelos autores

\section{RESULTADOS}

Nas tabelas a seguir são apresentados os resultados sumarizados dos indicadores de input e output para todo o país, conforme a pesquisa realizada. Em relação aos insumos, os dados são apresentados como proporção do conjunto de medicamentos, materiais e recursos humanos (equipe) completos com os quais as equipes contam, bem como a média da população atendida pelas. De forma geral, a média de existência de medicamentos e materiais necessários para o atendimento de hipertensão é maior, em relação ao diabetes.

De forma semelhante, os resultados para os produtos representam a proporção de equipes que realizam os procedimentos avaliados. Os dados mostram que a média para a realização dos procedimentos, tanto de hipertensão quanto de diabetes é próxima, com exceção da glicemia capilar de rotina, que possui média inferior aos demais processos e desvio padrão elevado, demonstrando que há grande variabilidade na realização deste procedimento entre as equipes avaliadas.

Quanto à eficiência, as médias dos escores dos estados brasileiros quanto ao cuidado de hipertensão e de diabetes, apresentados na Tabela 3, destacam uma distribuição homogênea dentro de cada grupo, sendo que a eficiência média do país para hipertensão situa-se em 63,95 e a de diabetes tem escore de 52,16. Os escores de eficiência relacionados à atenção à hipertensão foram superiores àqueles referentes ao cuidado do diabetes, revelando maior cumprimento, por parte das equipes, de ações destinadas ao cuidado de hipertensão, dados os insumos disponíveis.

O menor índice de eficiência em Hipertensão pertence a uma equipe do estado do Pará, e em Diabetes encontra-se em Pernambuco. É relevante 
destacar que em todos os estados existem equipes eficientes, ou seja, que são benchmarkings para as demais, o que se mostra importante na medida em que as equipes têm a possibilidade de buscar aumento de sua eficiência observando outras que atuam em um contexto semelhante.

Tabela 1: Média e desvio padrão das entradas Brasil, 2009.

\begin{tabular}{cccc}
\hline \multicolumn{4}{c}{ Hipertensão } \\
\hline Atendimento & Medicamentos & Materiais & $\begin{array}{c}\text { Recursos } \\
\text { Humanos }\end{array}$ \\
\hline Mean (dp) & Mean (dp) & Mean (dp) & Mean (dp) \\
\hline $3475(1549)$ & $0,64(0,31)$ & $0,96(0,10)$ & $0,90(0,30)$ \\
\hline \multicolumn{4}{c}{ Diabetes } \\
\hline Mean (dp) & Mean (dp) & Mean (dp) & Mean (dp) \\
\hline $3479(1551)$ & $0,55(0,32)$ & $0,72(0,22)$ & $0,90(0,29)$ \\
\hline
\end{tabular}

\section{Fonte: elaborado pelos autores}

Em relação às médias de eficiência encontradas, existem diferenças estatisticamente significativas entre os escores de hipertensão e diabetes dentro de uma mesma região, porém não se pode afirmar que existem diferenças na comparação entre as regiões, conforme teste de Chi-Square, nem para diabetes nem para hipertensão.

Tabela 2: Média e desvio padrão dos produtos Brasil, 2009.

\begin{tabular}{ccc}
\hline \multicolumn{3}{c}{ Hipertensão } \\
\hline Consultas & Acompanhamento & Registro Hiperdia \\
\hline Mean (dp) & Mean $(\mathrm{dp})$ & Mean $(\mathrm{dp})$ \\
\hline $0,98(0,13)$ & $0,98(0,14)$ & $0,93(0,25)$ \\
\hline \multicolumn{3}{c}{ Diabetes } \\
\hline Mean $(\mathrm{dp})$ & Mean $(\mathrm{dp})$ & Mean $(\mathrm{dp})$ \\
\hline $0,98(0,13)$ & $0,58(0,49)$ & $0,98(0,14)$ \\
\hline
\end{tabular}

Fonte: elaborado pelos autores

A figura 1 abaixo permite evidenciar que a quantidade absoluta de equipes eficientes nos estados quanto à oferta de cuidado de hipertensão e diabetes não variou substancialmente. A maior parte dos estados possui entre $5 \%$ e $15 \%$ de equipes eficientes, percentual que pode ser considerado baixo e que mantém relação com a qualidade dos serviços de saúde dedicados a atender condições crônicas, frente aos insumos disponíveis. A grande dispersão dos percentuais de eficiência nos estados evidencia a heterogeneidade nas regiões.

Ainda sobre a figura 1, quando feita a comparação entre os resultados de hipertensão e diabetes, conclui-se que são ações independentes, ou seja, a eficiência em uma não remetia à eficiência na outra, como pode ser observado.

\section{CONSIDERAÇÕES FINAIS}

Segundo relatório de 2005 da Organização Mundial de Saúde estima-se que a perda de renda nacional devido às doenças cardiovasculares e diabetes atingirão, nos próximos dez anos, 49,2 bilhões de dólares no Brasil, tendo em vista que aproximadamente $60 \%$ de todas as mortes são devidas às condições crônicas, em especial as doenças cardiovasculares, câncer e diabetes. (5). Estes dados enfatizam a importância indiscutível que a abordagem do cuidado de doenças crônicas representa para a saúde no país.

A oferta de serviços de saúde eficazes em relação ao diabetes e à hipertensão, com ênfase em ações de prevenção e controle, mais que em ações voltadas para episódios agudos, é capaz de reduzir os danos econômicos oriundos de complicações inerentes a estas doenças, bem como possibilitar uma maior qualidade de vida à população.

No entanto, a análise na abordagem de hipertensão e diabetes desenvolvida revelou uma situação de baixa eficiência. De maneira geral, os escores brasileiros situaram-se pouco acima de 50, evidenciando uma quantidade elevada de equipes que não cumprem as atividades mínimas para uma abordagem satisfatória destas doenças. A eficiência do cuidado da hipertensão arterial revelou-se ligeiramente superior, mas, ainda sim, em um patamar próximo a 60 , sinalizando a necessidade de intervenções para a promoção de melhoria nestes índices, dada a relevância destas ações. 
Tabela 3: Dados descritivos sobre a eficiência do tratamento da hipertensão e diabetes por estados da federação e por região administrativa. Brasil, 2009.

\begin{tabular}{|c|c|c|c|c|c|c|c|c|c|}
\hline \multirow{3}{*}{ Região } & \multirow[b]{3}{*}{ Estado } & \multirow{2}{*}{\multicolumn{4}{|c|}{ Hipertensão }} & \multirow{2}{*}{\multicolumn{4}{|c|}{ Diabetes }} \\
\hline & & & & & & & & & \\
\hline & & DMU's & Min & Média & \%Eficientes & DMU's & Min & Média & $\begin{array}{c}\% \\
\text { Eficientes } \\
\end{array}$ \\
\hline \multirow{5}{*}{$\begin{array}{l}\text { Centro- } \\
\text { Oeste }\end{array}$} & Distrito Federal & 17 & 41,68 & 65,27 & $11,76 \%$ & 17 & 29,07 & 48,81 & $11,76 \%$ \\
\hline & Goiás & 71 & 41,68 & 66,02 & $15,49 \%$ & 69 & 20,61 & 49,73 & $10,14 \%$ \\
\hline & Mato Grosso & 67 & 42,99 & 67,59 & $5,97 \%$ & 67 & 25,51 & 60,26 & $8,20 \%$ \\
\hline & $\begin{array}{l}\text { Mato Grosso do } \\
\text { Sul }\end{array}$ & 60 & 42,42 & 67,01 & $6,67 \%$ & 61 & 26,01 & 49,72 & $7,46 \%$ \\
\hline & Média da região & 215 & 41,68 & 66,47 & $9,77 \%$ & 214 & 20,61 & 52,13 & $8,88 \%$ \\
\hline \multirow{10}{*}{ Nordeste } & Alagoas & 60 & 42,08 & 66,51 & $3,33 \%$ & 60 & 24,69 & 56,17 & $3,33 \%$ \\
\hline & Bahia & 76 & 42,98 & 58,74 & $7,89 \%$ & 76 & 23,09 & 44,60 & $7,89 \%$ \\
\hline & Ceará & 58 & 41,68 & 64,12 & $18,97 \%$ & 57 & 21,75 & 54,46 & $17,54 \%$ \\
\hline & Maranhão & 53 & 45,11 & 62,66 & $13,21 \%$ & 52 & 27,67 & 53,94 & $15,38 \%$ \\
\hline & Paraíba & 57 & 41,68 & 60,54 & $8,77 \%$ & 56 & 26,29 & 56,07 & $8,93 \%$ \\
\hline & Pernambuco & 62 & 41,68 & 63,04 & $14,52 \%$ & 62 & 18,20 & 53,65 & $17,74 \%$ \\
\hline & Piauí & 52 & 43,09 & 57,82 & $1,92 \%$ & 52 & 24,60 & 42,88 & $5,77 \%$ \\
\hline & $\begin{array}{l}\text { Rio Grande do } \\
\text { Norte }\end{array}$ & 40 & 43,23 & 66,83 & $10,00 \%$ & 40 & 23,01 & 61,49 & $22,50 \%$ \\
\hline & Sergipe & 49 & 45,43 & 66,19 & $16,33 \%$ & 49 & 25,14 & 54,43 & $18,37 \%$ \\
\hline & Média da região & 507 & 41,68 & 62,94 & $10,45 \%$ & 504 & 18,20 & 53,08 & $12,50 \%$ \\
\hline \multirow{8}{*}{ Norte } & Acre & 53 & 41,68 & 64,96 & $15,09 \%$ & 52 & 19,28 & 52,30 & $13,46 \%$ \\
\hline & Amapá & 17 & 44,14 & 58,66 & $5,88 \%$ & 17 & 29,88 & 46,88 & $12,90 \%$ \\
\hline & Amazonas & 62 & 41,68 & 59,87 & $12,90 \%$ & 62 & 25,00 & 49,45 & $5,88 \%$ \\
\hline & Pará & 59 & 31,29 & 56,68 & $8,47 \%$ & 59 & 25,19 & 48,89 & $10,17 \%$ \\
\hline & Rondônia & 32 & 41,68 & 55,34 & $9,38 \%$ & 31 & 20,45 & 47,07 & $12,90 \%$ \\
\hline & Roraima & 32 & 42,88 & 63,92 & $21,88 \%$ & 32 & 27,36 & 60,88 & $21,88 \%$ \\
\hline & Tocantins & 45 & 44,17 & 66,22 & $11,11 \%$ & 45 & 25,83 & 57,60 & $17,78 \%$ \\
\hline & Média da região & 300 & 31,29 & 60,81 & $12,33 \%$ & 298 & 19,28 & 51,87 & $13,76 \%$ \\
\hline \multirow{5}{*}{ Sudeste } & Espírito Santo & 68 & 45,43 & 63,37 & $17,65 \%$ & 67 & 26,23 & 51,52 & $14,93 \%$ \\
\hline & Minas Gerais & 85 & 45,54 & 71,51 & $11,76 \%$ & 85 & 19,63 & 55,47 & $10,59 \%$ \\
\hline & Rio de Janeiro & 69 & 42,08 & 67,04 & $17,39 \%$ & 66 & 22,50 & 53,29 & $16,67 \%$ \\
\hline & São Paulo & 65 & 42,08 & 61,71 & $6,15 \%$ & 65 & 24,01 & 43,30 & $7,69 \%$ \\
\hline & Média da região & 287 & 42,08 & 65,90 & $13,24 \%$ & 283 & 19,63 & 50,90 & $12,37 \%$ \\
\hline \multirow{5}{*}{ Sul } & Paraná & 74 & 42,08 & 66,73 & $9,46 \%$ & 74 & 20,01 & 54,45 & $10,81 \%$ \\
\hline & $\begin{array}{l}\text { Rio Grande do } \\
\text { Sul }\end{array}$ & 77 & 42,88 & 65,30 & $11,69 \%$ & 77 & 19,00 & 50,42 & $14,29 \%$ \\
\hline & Santa Catarina & 72 & 41,68 & 63,32 & $2,78 \%$ & 72 & 20,28 & 51,23 & $9,72 \%$ \\
\hline & Média da região & 223 & 41,68 & 65,12 & $8,07 \%$ & 223 & 19,00 & 52,03 & $11,66 \%$ \\
\hline & BRASIL & 1532 & 31,29 & 63,95 & $10,90 \%$ & 1522 & 18,20 & 52,16 & $12,09 \%$ \\
\hline
\end{tabular}

Fonte: elaborado pelos autores 
Figura 1: Mapa comparativo dos percentuais de equipamentos que são eficientes em hipertensão e diabetes por estado. Brasil, 2009

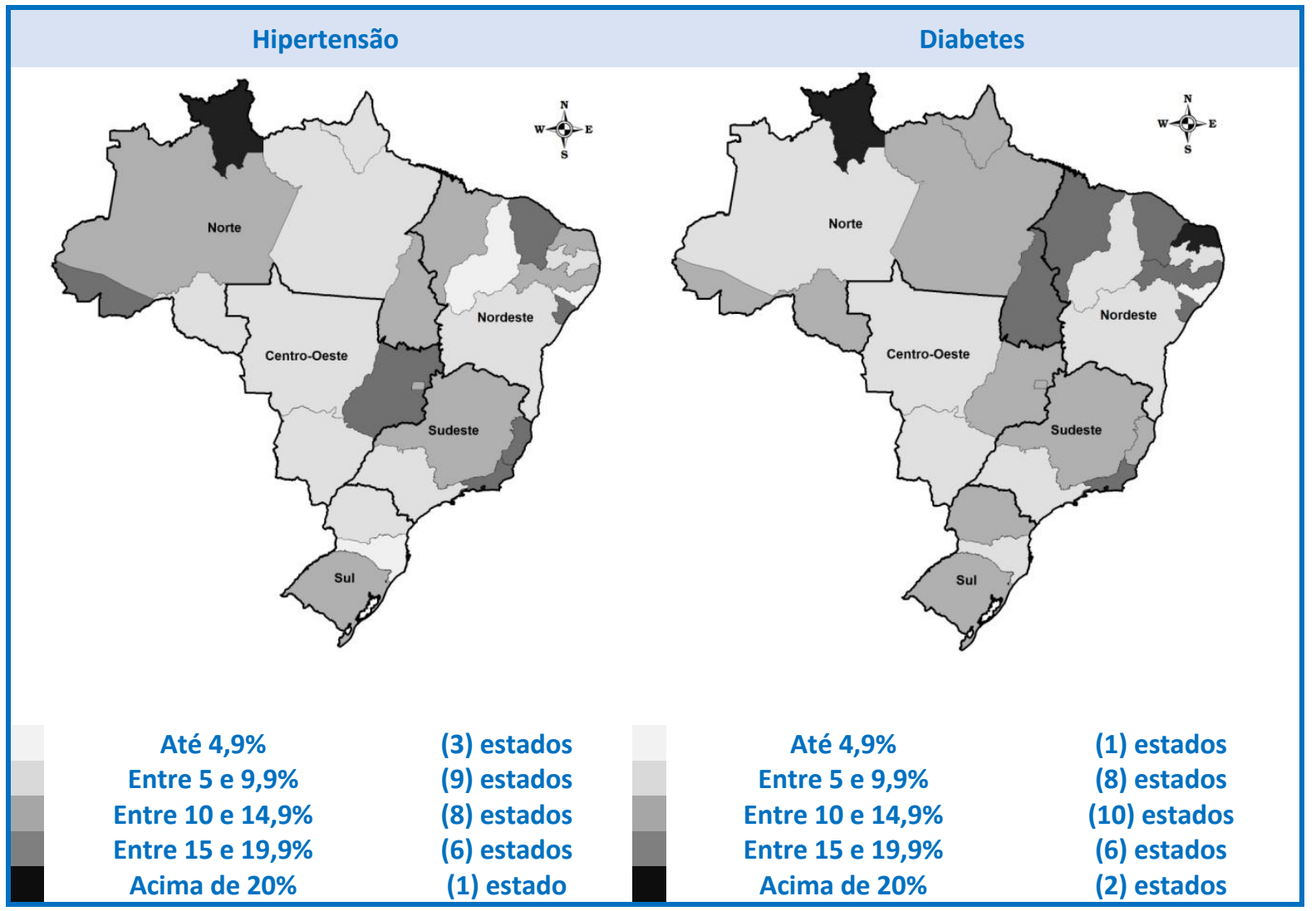

Fonte: elaborado pelos autores

O fato das equipes de saúde terem demonstrado eficiência superior no cuidado de hipertensão em relação ao cuidado de diabetes pode ser reflexo, em parte, da situação geral observada nas equipes avaliadas, uma vez que a proporção média da disponibilidade de materiais e medicamentos é também superior para a primeira. A análise desta relação ressalta de forma inequívoca a importância dos elementos de estrutura no cumprimento do processo de trabalho e, em última instância, no desempenho das equipes de Saúde da Família.

A dimensão do processo de trabalho, por sua vez, mostra-se também essencial para a melhoria do desempenho das equipes. No que se refere ao cuidado em diabetes, os resultados indicam a necessidade de aumento na execução de ações de prevenção e controle, fundamentais para uma abordagem eficiente desta condição crônica, destacada pela necessidade de aumento na realizado de glicemia capilar para assintomáticos, seguida pela necessidade de aumento na realização de consultas para diagnóstico. Um incremento no nível de execução destas duas atividades melhoraria a eficiência das equipes.

Para a hipertensão, a melhoria da eficiência está condicionada ao aumento do cadastro de pacientes no sistema próprio de monitoramento e acompanhamento, o que revela a necessidade de aumento do volume das informações que subsidiam o desenvolvimento de políticas para o cuidado desta doença.

Assim, a necessidade de investimentos, tanto em estrutura, por meio da aquisição de equipamentos imprescindíveis para a realização das atividades, quanto em formação e capacitação das equipes, no que diz respeito ao processo de trabalho, conforme o protocolo estabelecido, revelam-se como 
possíveis vias para a busca de aumento da eficiência no cuidado ofertado para estas doenças crônicas.

Por fim, entende-se que o aumento da eficiência neste caso, além de possibilitar melhor oferta de cuidados à população, representa eficiência na utilização dos recursos materiais e financeiros, o que se torna imprescindível no contexto das políticas públicas. Neste sentido, vale destacar que as ações de intervenção no nível da atenção primária impactam de forma expressiva o sistema de saúde como um todo, pois evitam agravos que sobrecarregam os demais níveis de atenção nos quais há maior necessidade de recursos e, por isso, maiores custos, além de maior risco para os pacientes. Assim, os resultados obtidos com a utilização da metodologia de DEA assumem grande relevância ao ressaltar a necessidade de maior investimento para que este nível de atenção à saúde cumpra seu papel na organização da rede de saúde.

\section{REFERÊNCIAS BIBLIOGRÁFICAS}

(1) INSTITUTO BRASILEIRO DE GEOGRAFIA E ESTATÍSTICA (IBGE). Projeção da população do Brasil por sexo e idade para o período 1980-2050; Brasil: 2004.

(2) PRATA, Pedro Reginaldo. The Epidemiologic Transition in Brazil. Cadernos de Saúde Pública, Rio de Janeiro, 8 (2): 168-175, abr/jun, 1992.

(3) OMRAN, Abdel R. The Epidemiologic Transition: a Theory of the Epidemiology of Population Change. Extract From The Milbank Memorial Fund Quartely 1971, 49(4): 509-538. Bulletin of The World Health Organization, 79(2).

(4) Ministério da Saúde do Brasil. Secretaria de Atenção à Saúde. Departamento de Atenção Básica. Diabetes Mellitus: Cadernos de Atenção Básica, n. 16. 64 p. Brasil: 2006.

(5) WORLD HEALTH ORGANIZATION. Preventing chronic diseases: a vital investment. Geneva: WHO/Public Health Agency of Canada; 2005.
(6) Ministério da Saúde do Brasil. Plano de reorganização da atenção à hipertensão arterial e ao diabetes mellitus: hipertensão arterial e diabetes mellitus, 104p. Brasil: 2001.

(7) STARFIELD, Barbara. Atenção primária: equilíbrio entre necessidades de saúde, serviços e tecnologia. Brasília: UNESCO, Ministério da Saúde; 2002.

(8) MENDES, Eugênio Vilaça. A atenção primária à saúde no SUS. Fortaleza: Escola de Saúde Pública do Ceará; 2002.

(9) ACHUTTI, Aloyzio \& AZAMBUJA, Maria Inês Reinert. Doenças crônicas não-transmissíveis no Brasil: repercussões do modelo de atenção à saúde sobre a seguridade social. Ciência \& Saúde coletiva [online], vol.9, n.4, pp. 833-840. Brasil: 2004.

(10) LESSA, Inês. et al. Simultaneidade de fatores de risco cardiovascular modificáveis na população adulta de Salvador. Revista Panamericana de Salud Pública 16:131-137; 2004.

(11) Ministério da Saúde do Brasil. Diretrizes e recomendações para o cuidado integral de doenças crônicas não-transmissíveis: promoção da saúde, vigilância, prevenção e assistência - Brasília: Ministério da Saúde, 2008. 72 p. - (Série Pactos pela Saúde 2006; v. 8)

(12) Ministério da Saúde do Brasil. Saúde da Família: Uma Estratégia para a Reorientação do Modelo Assistencial. Brasil: 1997.

(13) BRASIL. Portaria 648 de 5 de dezembro de 2006. Política Nacional de Atenção Básica. Diário Oficial da República Federativa do Brasil, Brasília; 2006.

(14) COLIN, Emerson. Pesquisa Operacional: Aplicações em Estratégia, Finanças, Logística,Produção, Marketing e Vendas. Rio de Janeiros: LTC; 2007.

(15) COOK, W.D. and ZHU, Joe. Data Envelopment Analysis: Modeling Operational Processes And Measuring Productivity. 2008

(16) SOARES DE MELLO, J.C.C. B.; Angulo Meza, L.; Gomes, E.G. e Biondi Neto, L. Curso de Análise de Envoltória de Dados. Anais do XXXVII Simpósio 
Brasileiro de Pesquisa Operacional, SBPO, Gramado; 2005.

(17) AMADO, Carla Alexandra da Encarnação Filipe e SANTOS, Sérgio Pereira dos. Challenges for performance assessment and improvement in primary health care: The case of the Portuguese health centres. Health Policy 91 43-56; 2009.

(18) DONABEDIAN, A. Basic approaches to assessment: structure, process and outcome. In: Explorations in Quality Assessment and Monitoring. Michigan: Health Administration Press vol. I, p.77$125 ; 1980$.
(19) DONABEDIAN, A. The role of outcomes in quality assessment and assurance. Quality Review Bulletin, 18: 356-360; 1992.

(20) OPAS/OMS. Disponível em: http://portalweb02.saude.gov.br/saude/ visualizar texto.cfm?idtxt $=17098$ Acesso em 14/03/2004.

(21) ORGANIZAÇÃO MUNDIAL DA SAÚDE (OMS). Preventing Chronic Diseases a vital investments. Geneva, 2005. 182 p.

\section{ABSTRACT}

Introduction: This work therefore aims to comparatively analyze the efficiency of Family Health teams in the different Brazilian states regarding the prevention, treatment and follow - up of hypertension and diabetes mellitus. From the result it will be possible to outline the actions that must be prioritized, so that the teams become efficient in the processes fundamental to the care related to these diseases.

Method: The evaluation of the efficiency of the Family Health teams was elaborated using the Data Envelopment Analysis (DEA) methodology. DEA that uses the linear programming technique to calculate the relative efficiency of a set of productive units that have common function and operate in a similar way, but whose efficiency varies according to internal aspects. For this work, we opted for the output-oriented CRS model to evaluate the efficiency of family health teams (DMUs). In all were assessed 2133 family health teams, sample is set with a maximum error of $2 \%$.

Results: Most states have between $\mathbf{5 \%}$ and $\mathbf{1 5 \%}$ of efficient teams, a percentage that can be considered low and that is related to the quality of health services dedicated to attending chronic conditions, compared to the available inputs. The large dispersion of the efficiency percentages in the states shows the heterogeneity in the regions

Conclusions: Finally, it is understood that increasing efficiency in this case, in addition to providing a better offer of care to the population, represents efficiency in the use of material and financial resources, which becomes essential in the context of public policies. In this sense, it is worth noting that intervention actions at the primary health care level significantly impact the health system as a whole, since they avoid diseases that burden the other levels of care in which there is a greater need for resources and, therefore, greater costs, in addition to greater risk for patients. Thus, the results obtained with the use of the DEA methodology assume great importance in emphasizing the need for greater investment so that this level of health care fulfills its role in the organization of the health network.

Keywords: Operational research, health assistance, chronical disease. 